\title{
An attitudinal consensus degree to control feedback mechanism in group decision making with different adjustment cost
}

\author{
Jian $\mathrm{Wu}^{\mathrm{a}}$, Qi Sun ${ }^{\mathrm{a}}$, Hamido Fujita ${ }^{\mathrm{b}}$, Francisco Chiclana ${ }^{\mathrm{c}, \mathrm{d}}$ \\ ${ }^{a}$ School of Economics and Management, Shanghai Maritime University, Shanghai, China \\ ${ }^{b}$ Iwate Prefectural University, Takizawa, Iwate, Japan \\ ${ }^{c}$ Institute of Artificial Intelligence, Faculty of Computing, Engineering and Media, De Montfort University, Leicester, \\ $U K$ \\ ${ }^{d}$ Department of Computer Science and Artificial Intelligence, University of Granada, Spain
}

\begin{abstract}
This article aims to study the influence of the group attitude on the consensus reaching process in group decision making (GDM). To do that, the attitudinal consensus index (ACI) is defined to aggregate individual consensus levels to form a a collective one. This approach allows for the implementation of the group attitude in a continuous state ranging from a pessimistic attitude to an optimistic attitude. Then, ACI is used to build a stop policy to control feedback for consensus, which can be regarded as a generation of the traditional polices: 'minimum disagreement policy' and 'indifferent disagreement policy'. A sensitivity analysis method with visual simulation is proposed to check the adjustment cost and consensus level with different attitudinal parameters. The main conclusion from this analysis is that the bigger the attitudinal parameter implemented is, the bigger the adjustment cost and consensus level are. The visual information facilitates the inconsistent expert keeping a balance between the attitudinal parameter to implement and the adjustment cost and consensus level, which in practice translates into full control of such implementation based on the decision maker's willingness.
\end{abstract}

Keywords: Group decision making, Consensus, Attitude, Adjustment cost

\section{Introduction}

In group decision making (GDM), experts express their preferences on a finite set of alternatives $X=\left\{x_{1}, \ldots, x_{n}\right\}$ and then aggregate them to produce a group ranking order of alternatives. A classic aggregation approach in this context is the 'majority rule' [18]. However, this rule did not take into account the consensus (agreement) level between the group of experts, which could lead to some of them to reject the final decision result because their individual preferences were not appropriately considered in the resolution process [28]. In realistic decision contexts, it is rare for a group of experts to have consensus (agreement) before the aforementioned aggregation step due to their different background and knowledge on the decision problem; thus, it is worth having mechanisms to support experts to assess their consensus and subsequently to reach consensus before the aggregation of their preferences

Email addresses: jyajian@163.com (Jian Wu), qisun1207@126.com (Qi Sun), HFujita-799@acm.org (Hamido Fujita), chiclana@dmu.ac.uk; inv.chiclana@ugr.es (Francisco Chiclana) 
$[3,10,13,14,17,19,27,35,43]$. This type of consensus mechanism, which is called consensus reaching process $(\mathrm{CRP})$, is regarded as an effective methodology to reduce or eliminate inconsistency in GDM because they usually involved the application of a feedback process that: (1) identified the experts who are inconsistent with the group and (ii) generated recommendation advices to support them in reducing their inconsistency with the group of experts [6, 17, 20, 23, 31, 32]. In a CRP, the consensus level of the group of experts is monitored to judge whether or not to implement the feedback process $[4,5,7,15,29,37]$, and a stop policy is also in place to determine when the consensus process needs to come to an end.

The stopping policy procedure is a key element in the feedback mechanism of the CRP. The stopping policy mostly used in CRPs first aggregates individual consensus levels to derive the group consensus level. If the group consensus level is lower than an acceptable consensus threshold value, then the feedback mechanism is activated; otherwise the resolution process of the GDM is carried out $[9,11,12,24,26]$. For example, the 'minimum disagreement policy' [34] used the minimum operator in aggregating individual consensus levels, which in practice forces all experts in the group to reach the consensus threshold value before the resolution process can be activated. The 'indifferent disagreement policy' [41] used the arithmetic mean to aggregate individual consensus levels. It is clear that the choice of the aggregation operator to implement in the feedback process of a CRP has a direct consequence on the number of interaction rounds before group consensus reaches the consensus threshold value. As such, the 'minimum disagreement policy' can be seen as stricter than the 'indifferent disagreement policy' because the latter is a compensatory aggregation operator while the former is not. Nevertheless, both the above stopping policies are examples of policies that do not take into account the attitude of the group, which can affect both the number of interaction rounds and the adjustment cost of the feedback process, i.e. the efficiency of the CRP. Thus, the attitude of the group, which has been regarded as an important issue in GDM, is often neglected in the CRP.

Recently, Yager [41] presented a very interesting idea for stopping policy that can reflect the attitude of a group, which is based on the aggregation of the individual consensus levels by the order weighted average (OWA) operator. Notice that the OWA operator contains the minimum operator and the arithmetic mean as special cases, thus Yager's 'OWA based policy' [41] is a more general stopping policy formulation than the 'minimum disagreement policy' and the 'indifferent disagreement policy'. Palomares et al. [25] also regarded the CRP to be affected by the attitude and introduced three types of consensus: 'pessimistic', 'indifferent' and 'optimistic'. Inspired by these interesting ideas, this article aims to investigate an attitudinal consensus index (ACI) for feedback in CRP. The advantage of the proposed ACI is that it takes into account group attitudes in a continuous state from pessimistic to optimistic, and then it can be regarded as an extension of both Yager's 'OWA based policy' [41] and the 'three types of consensus policy' by Palomares et al. [25]. Based on the ACI, a sensitivity analysis method for adjustment cost with different attitudinal parameter is investigated and the following 
result is proved: the adjustment cost increases as the attitudinal parameter decreases. At the same time, the consensus level of inconsistent experts increases after each feedback, which guarantees the convergence of the CRP. A visual simulation of the increase of adjustment cost and consensus level after each feedback process round is designed to support the inconsistent experts in selecting an appropriate attitudinal parameter to keep a balance between their adjustment cost and consensus level, which in practice will facilitate the acceptance of the final decision results by the individual experts as 'the outcome' of their own willing implementation of the recommendation advice provided by the feedback process.

The rest of paper is set out as follows: In Section 2, the concept of the attitudinal consensus index (ACI) is defined for the case of preferences being in the form of interval-valued intuitionistic fuzzy preference relations (IVIFPRs), which are defined in Section 2.1. Then, an ACI based consensus reaching process model is proposed. In Section 3, the ACI is used to construct a stopping policy to control the feedback mechanism. In addition, Section 4 calculates the feedback interaction costs and analyses the its relationship with the attitude value. Some comparison analysis with other approaches are conducted in Section 5. Finally, conclusions are drawn in Section 6.

\section{An attitude based consensus model in group decision making}

As aforementioned, inconsistency in the CRP might happen due to the diverse preferences of experts. Nevertheless, it is preferable that the group of experts reach consensus before aggregating individual preferences into a collective one. To do so, this article introduces the proximity degrees (PD) at three levels: (1) elements level; (2) alternatives level; and (3) preference relation (decision matrix) level. If all the decision matrix level PDs (group PD) are above a fixed threshold value of group consensus, then the resolution process of the GDM is activated; otherwise the feedback mechanism is applied to identify the inconsistent experts and generate recommendation advices to increase the current group consensus level. However, this traditional policy requires each expert reach the threshold value $\gamma$, and then it is a strict policy. Therefore, this article proposes a new stop policy by investigating an attitudinal consensus degree with different adjustment cost. To do this, the adjustment cost for reaching consensus based on the attitude of experts is calculated and this information is passed to the inconsistent users in order to empower them with the know-how to adopt the recommendation advices if they are willing to reach the threshold value of attitudinal consensus degree. The present paper assumes that opinions are provided in the form of interval-valued intuitionistic fuzzy decision matrices (IVIFDMs), which are introduced below.

\subsection{Preliminaries}

The intuitionistic fuzzy set (IFS) and interval-valued fuzzy set (IVFS) are mathematically equivalent [8], and so the intuitionistic fuzzy decision matrix (IFDM) and interval-valued fuzzy decision 
matrix (IVFDM) are [30]. This result was subsequently exploited by Wu et al. in [33] to derive the correct theoretical formulation of the multiplicative transitivity property. Consequently, IFSs and IVFSs are completely equivalent in MCDM resolution processes, and then hesitation and uncertainty can be unified.

The concept of interval-valued intuitionistic fuzzy set (IVFS) was introduced by Atanassov and Gargov [1]:

Definition 1 (Interval-Valued Intuitionistic Fuzzy Set (IVIFS)). "Let $\operatorname{INT}([0,1])$ be the set of all closed subintervals of the unit interval and $X$ be a universe of discourse. An interval-valued IFS (IVIFS) $A$ over $X$ is given as:

$$
A=\left\{\left\langle x, \tilde{\mu}_{A}(x), \tilde{\nu}_{A}(x)\right\rangle \mid x \in X\right\}
$$

where $\tilde{\mu}_{A}(x), \tilde{\nu}_{A}(x) \in \operatorname{INT}([0,1])$, represent the membership and the non-membership degrees of the element $x$ to the set $A$ subject to the constraint $0 \leq \sup \tilde{\mu}_{A}(x)+\sup \tilde{\nu}_{A}(x) \leq 1, \forall x \in X$.

Denoting by $\tilde{\mu}_{A L}(x), \tilde{\mu}_{A U}(x)$ and $\tilde{\nu}_{A L}(x), \tilde{\nu}_{A U}(x)$ the lower and upper end points of $\tilde{\mu}_{A}(x)$ and $\tilde{\nu}_{A}(x)$, respectively, an IVIFS can be represented as $A=\left\{\left\langle x,\left[\tilde{\mu}_{A L}(x), \tilde{\mu}_{A U}(x)\right],\left[\tilde{\nu}_{A L}(x), \tilde{\nu}_{A U}(x)\right]\right\rangle \mid x \in\right.$ $\left.\left.X: 0 \leq \tilde{\mu}_{A U}(x)+\tilde{\nu}_{A U}(x)\right) \leq 1, \tilde{\mu}_{A L}(x) \wedge \tilde{\nu}_{A L}(x) \geq 0\right\}$. The hesitancy degree function of an IVIFS is $\tilde{\pi}_{A}(x)=\left[1-\tilde{\mu}_{A U}(x)-\tilde{\nu}_{A U}(x), 1-\tilde{\mu}_{A L}(x)-\tilde{\nu}_{A L}(x)\right] . "$

The concept of interval-valued intuitionistic fuzzy decision matrix (IVIFDM) derives from the the definition of interval-valued fuzzy number as a generalisation of the concept of fuzzy decision matrix [33]. Indeed, a matrix $A=\left(\tilde{a}_{i j}\right)_{m \times n}$ with IVIFN elements $\tilde{a}_{i j}$ will be called an IVIFDM. Because the aforementioned isomorphism between intuitionists and interval-valued fuzzy sets, the operational laws of the latter will be implemented herein.

\subsection{Similarity degrees}

Based on the Hamming distance, a distance function between IVIFNs can be defined [39]:

Definition 2 (IVIFNs distance). "The normalised Hamming distance between two IVIFNs is

$$
d\left(\tilde{\alpha}_{1}, \tilde{\alpha}_{2}\right)=\frac{1}{4}\left(\left|\mu_{1}^{-}-\mu_{2}^{-}\right|+\left|\mu_{1}^{+}-\mu_{2}^{+}\right|+\left|v_{1}^{-}-v_{2}^{-}\right|+\left|v_{1}^{+}-v_{2}^{+}\right|\right)
$$

where $\tilde{\alpha}_{1}=\left(\left[\mu_{1}^{-}, \mu_{1}^{+}\right],\left[\nu_{1}^{-}, \nu_{1}^{+}\right]\right)$and $\tilde{\alpha}_{2}=\left(\left[\mu_{2}^{-}, \mu_{2}^{+}\right],\left[\nu_{2}^{-}, \nu_{2}^{+}\right]\right) . "$

$\mathrm{Xu}$ and Yager [39] defined the similarity degree between IVIFNs for consensus analysis in GDM.

Definition 3 (Similarity degree). "The similarity degree of two IVIFNs is

$$
\theta\left(\tilde{\alpha}_{1}, \tilde{\alpha}_{2}\right)=\left\{\begin{array}{l}
0.5, \quad \tilde{\alpha}_{1}=\tilde{\alpha}_{2}=\overline{\alpha_{2}} ; \\
\frac{d\left(\tilde{\alpha}_{1}, \overline{\alpha_{2}}\right)}{d\left(\tilde{\alpha}_{1}, \tilde{\alpha}_{2}\right)+d\left(\tilde{\alpha}_{1}, \overline{\alpha_{2}}\right)}, \text { otherwise }
\end{array}\right.
$$

where $\tilde{\alpha}_{1}=\left(\left[\mu_{1}^{-}, \mu_{1}^{+}\right],\left[\nu_{1}^{-}, \nu_{1}^{+}\right]\right)$and $\tilde{\alpha}_{2}=\left(\left[\mu_{2}^{-}, \mu_{2}^{+}\right],\left[\nu_{2}^{-}, \nu_{2}^{+}\right]\right)$and $\overline{\tilde{\alpha}_{2}}=\left(\left[\nu_{2}^{-}, \nu_{2}^{+}\right],\left[\mu_{2}^{-}, \mu_{2}^{+}\right]\right)$is the complementary IVIFN of $\tilde{\alpha}_{2} . "$ 


\subsection{Proximity Degree}

Once the collective decision matrix is obtained, $A^{c}=\left(\tilde{a}_{i j}^{c}\right)$, by fusing the individual decision matrices, $A^{h}=\left(\tilde{a}_{i j}^{h}\right)$, the proximity degree $(\mathrm{PD})$ of each expert to the group can be computed at the three different levels of a relation:

Level 1. Proximity degree on elements of alternatives. The proximity degree of an expert $e_{h}$ to the group on the elements is calculated as:

$$
P E_{i j}^{h}=\theta\left(\tilde{a}_{i j}^{h}, \tilde{a}_{i j}^{c}\right)
$$

Level 2. Proximity degree on alternatives. The proximity degree of an expert $e_{h}$ to the group on the alternative is calculated as:

$$
P A_{i}^{h}=\frac{1}{n} \sum_{j=1}^{n} P E_{i j}^{h}
$$

Level 3. Proximity degree on preference of experts. The proximity degree of an expert $e_{h}$ to the group on the set of experts is calculated as:

$$
P D^{h}=\frac{1}{m} \sum_{i=1}^{m} P A_{i}^{h}
$$

Example 1. A home appliance company would like to select the most appropriate supplies for its purchasing target. After pre evaluation, four buyers $\left\{M_{1}, M_{2}, M_{3}, M_{4}\right\}$ have remained as alternatives for further evaluation. Three purchase criteria $\left\{N_{1}, N_{2}, N_{3}\right\}$ are considered as follows: $N_{1}$, technological level; $N_{2}$, product quality; $N_{3}$, manufacturability. Four experts $\left\{e_{1}, e_{2}, e_{3}, e_{4}\right\}$ from different departments, whose important degrees are the same, provide the following IVIFDMs:

$$
\begin{aligned}
& A^{1}=\left(\begin{array}{cccc}
N_{1} & N_{2} & N_{3} \\
M_{1} & \langle[0.2,0.3],[0.5,0.6]\rangle & \langle[0.3,0.4],[0.5,0.6]\rangle & \langle[0.1,0.2],[0.6,0.7]\rangle \\
M_{2} & \langle[0.3,0.4],[0.4,0.5]\rangle & \langle[0.5,0.6],[0.2,0.3]\rangle & \langle[0.6,0.7],[0.1,0.2]\rangle \\
M_{3} & \langle[0.2,0.3],[0.5,0.6]\rangle & \langle[0.1,0.2],[0.5,0.6]\rangle & \langle[0.2,0.4],[0.4,0.6]\rangle \\
M_{4} & \langle[0.3,0.4],[0.5,0.6]\rangle & \langle[0.5,0.6],[0.3,0.4]\rangle & \langle[0.1,0.2],[0.6,0.7]\rangle
\end{array}\right) \\
& A^{2}=\left(\begin{array}{cccc}
N_{1} & N_{2} & N_{3} \\
M_{1} & \langle[0.2,0.3],[0.6,0.7]\rangle & \langle[0.3,0.4],[0.5,0.6]\rangle & \langle[0.3,0.4],[0.5,0.6]\rangle \\
M_{2} & \langle[0.4,0.5],[0.3,0.5]\rangle & \langle[0.1,0.2],[0.7,0.8]\rangle & \langle[0.5,0.6],[0.2,0.3]\rangle \\
M_{3} & \langle[0.2,0.4],[0.5,0.6]\rangle & \langle[0.3,0.4],[0.5,0.6]\rangle & \langle[0.2,0.3],[0.5,0.6]\rangle \\
M_{4} & \langle[0.2,0.3],[0.6,0.7]\rangle & \langle[0.4,0.5],[0.2,0.3]\rangle & \langle[0.4,0.5],[0.2,0.3]\rangle
\end{array}\right) \\
& A^{3}=\left(\begin{array}{cccc}
N_{1} & N_{2} & N_{3} \\
M_{1} & \langle[0.2,0.3],[0.3,0.4]\rangle & \langle[0.3,0.4],[0.4,0.5]\rangle & \langle[0.1,0.2],[0.4,0.5]\rangle \\
M_{2} & \langle[0.3,0.4],[0.3,0.4]\rangle & \langle[0.3,0.4],[0.4,0.5]\rangle & \langle[0.5,0.6],[0.4,0.4]\rangle \\
M_{3} & \langle[0.3,0.4],[0.5,0.6]\rangle & \langle[0.3,0.4],[0.3,0.4]\rangle & \langle[0.4,0.5],[0.5,0.5]\rangle \\
M_{4} & \langle[0.6,0.7],[0.2,0.3]\rangle & \langle[0.4,0.5],[0.1,0.3]\rangle & \langle[0.5,0.6],[0.3,0.4]\rangle
\end{array}\right)
\end{aligned}
$$




$$
A^{4}=\left(\begin{array}{cccc}
N_{1} & N_{2} & N_{3} \\
M_{1} & \langle[0.3,0.4],[0.5,0.6]\rangle & \langle[0.3,0.4],[0.5,0.6]\rangle & \langle[0.1,0.2],[0.5,0.6]\rangle \\
M_{2} & \langle[0.3,0.4],[0.4,0.5]\rangle & \langle[0.2,0.3],[0.5,0.6]\rangle & \langle[0.1,0.2],[0.6,0.7]\rangle \\
M_{3} & \langle[0.3,0.4],[0.5,0.6]\rangle & \langle[0.3,0.5],[0.4,0.5]\rangle & \langle[0.3,0.4],[0.5,0.6]\rangle \\
M_{4} & \langle[0.1,0.2],[0.3,0.4]\rangle & \langle[0.3,0.4],[0.5,0.6]\rangle & \langle[0.2,0.3],[0.4,0.6]\rangle
\end{array}\right)
$$

(1) The individual IVIFDMs are aggregated to derive the following collective IVIFDM:

$$
A^{c}=\left(\begin{array}{cccc}
N_{1} & N_{2} & N_{3} \\
M_{1} & \langle[0.23,0.33],[0.48,0.58]\rangle & \langle[0.30,0.40],[0.48,0.58]\rangle & \langle[0.15,0.25],[0.50,0.60]\rangle \\
M_{2} & \langle[0.33,0.43],[0.35,0.48]\rangle & \langle[0.28,0.38],[0.45,0.55]\rangle & \langle[0.43,0.53],[0.33,0.40]\rangle \\
M_{3} & \langle[0.25,0.38],[0.50,0.60]\rangle & \langle[0.25,0.38],[0.43,0.53]\rangle & \langle[0.28,0.40],[0.48,0.58]\rangle \\
M_{4} & \langle[0.30,0.40],[0.40,0.50]\rangle & \langle[0.40,0.50],[0.28,0.40]\rangle & \langle[0.30,0.40],[0.38,0.50]\rangle
\end{array}\right)
$$

(2) Proximity degrees computation.

Level 1. The proximity degree on elements of alternatives:

$$
\begin{aligned}
P E^{1} & =\left(\begin{array}{lll}
0.917 & 0.938 & 0.850 \\
0.688 & 0.208 & 0.613 \\
0.896 & 0.703 & 0.816 \\
0.750 & 0.735 & 0.588
\end{array}\right) P E^{2}=\left(\begin{array}{lll}
0.813 & 0.938 & 0.786 \\
0.438 & 0.646 & 0.688 \\
0.929 & 0.763 & 0.813 \\
0.625 & 0.781 & 0.281
\end{array}\right) \\
P E^{3} & =\left(\begin{array}{lll}
0.636 & 0.786 & 0.813 \\
0.500 & 0.786 & 0.700 \\
0.921 & 0.500 & 0.594 \\
0.375 & 0.725 & 0.281
\end{array}\right) P E^{4}=\left(\begin{array}{lll}
0.818 & 0.938 & 0.938 \\
0.688 & 0.792 & 0.388 \\
0.921 & 0.654 & 0.912 \\
0.500 & 0.265 & 0.675
\end{array}\right)
\end{aligned}
$$

Level 2. The proximity degree on alternatives:

$$
\begin{gathered}
P A^{1}=\left(\begin{array}{llll}
0.901, & 0.503, & 0.805, & 0.691
\end{array}\right) ; P A^{2}=\left(\begin{array}{llll}
0.845, & 0.590, & 0.835, & 0.563
\end{array}\right) ; \\
P A^{3}=\left(\begin{array}{llll}
0.745, & 0.662, & 0.672, & 0.460
\end{array}\right) ; P A^{4}=\left(\begin{array}{llll}
0.898, & 0.622, & 0.829, & 0.480
\end{array}\right)
\end{gathered}
$$

Level 3. The proximity degree on the preference of experts:

$$
P D^{1}=0.725 ; P D^{2}=0.708 ; P D^{3}=0.635 ; P D^{4}=0.707
$$




\subsection{Attitudinal Consensus Index}

In the group decision making problems, it is often difficult for experts to reach complete agreement. In these cases, a threshold value of group consensus $\gamma$, in the region $[0.5,1)$, is normally expected to be reached, and group interaction happens while group consensus is below it. Traditional consensus models $[3,17,31,43]$ usually used the value $\min \left(P D^{1}, \ldots, P D^{k}\right)$ as a stop policy to control the interaction process: 'If $\min \left(P D^{1}, \ldots, P D^{k}\right) \geq \gamma$, then the resolution process of the GDM is carried out; while if $\min \left(P D^{1}, \ldots, P D^{k}\right)<\gamma$, the feedback mechanism is activated to identify the inconsistent experts'. This policy is considered a 'strict' stop policy because it requires 'all' experts to reach the threshold value $\gamma$, and it is named the 'minimum disagreement policy'. This stop policy may involve quite a large of adjustment cost, specially when a high number of experts are involved in the GDM process. Alternative operators to the min operator are obvious choices to implement in the stop policy. For example, the 'indifferent disagreement policy' [41] implemented the arithmetic average operator to control the consensus reaching process (CRP). A more general formulation including these two stop policies is possible with implementing Yager's OWA operator Yager[41]. Additionally, Palomares et al. in [25] classified the attitude towards consensus as being: 'pessimistic', 'indifferent' and 'optimistic'.

Inspired by these interesting ideas, this article proposes an attitudinal consensus index (ACI) which takes into account decision attitudes in a continuous state from pessimistic to optimistic.

Definition 4 (Attitudinal Consensus Index (ACI)). Let $P D^{1}, \ldots, P D^{k}$ be a set of proximity degrees associated to a group experts. The value

$$
A C I=\sum_{h=1}^{k} \omega_{h} \times P D^{\sigma(h)}
$$

where $\sigma$ is the permutation such that $P D^{\sigma(h+1)} \leq P D^{\sigma(h)}(\forall h=1, \ldots, k-1)$, is called the attitudinal consensus index.

Notice that the ACI is the result of an OWA aggregation of the individual PDs, $\left(P D^{1}, \ldots, P D^{k}\right)$, and consequently the weights, $\left(\omega_{1}, \ldots, \omega_{k}\right)$, can be calculated using Yager's linguistic quantifier based approach [40]:

$$
\omega_{h}=Q\left(\frac{h}{n}\right)-Q\left(\frac{h-1}{n}\right)
$$

where $Q:[0,1] \rightarrow[0,1]$ is a basic unit-interval monotonic (BUM) mapping, i.e. an increasing function such that $Q(0)=0$ and $Q(1)=1$. In particular, Yager [40] introduced the parameterised family of regular increasing monotone (RIM) quantifiers $Q(x)=x^{r}(r \geq 0)$ for such representation. For example, when $r=1$ the 'indifferent disagreement policy' happens, while when $r \rightarrow+\infty$ the 'minimum disagreement policy' happens (pessimistic). When $r=0$, the stop policy based on the max operator ('maximum disagreement policy') would result (optimistic). Thus, by computing the weights assigned to $P D^{\sigma(h)}$ using a value $r$, representing the attitude of the group towards consensus, the proposed 
attitudinal consensus index (ACI) will in fact allow the implementation of the the group attitude towards consensus in general, and it will allow continuous transition from an optimistic to a pessimistic attitude towards consensus.

Example 2. (Example 1 continuation) Using the regular increasing monotone (RIM) quantifiers $Q(x)=x^{r}(r \geq 0)$, the weights $\omega_{h}(h=1,2,3,4)$ and the ACI representing the group attitudinal parameter to consensus are given in Table 1, which clearly reflects the aforementioned monotonic decreasing relationship of $A C I$ with respect to $r$.

\begin{tabular}{|c|c|c|c|c|c|c|c|c|c|}
\hline$r$ & 0 & $\frac{1}{3}$ & $\frac{1}{2}$ & $\frac{2}{3}$ & 1 & $\frac{3}{2}$ & 2 & $\ldots$ & $+\infty$ \\
\hline$\omega_{1}$ & 1 & 0.630 & 0.500 & 0.397 & 0.250 & 0.125 & 0.063 & & 0 \\
\hline$\omega_{2}$ & 0 & 0.164 & 0.207 & 0.233 & 0.250 & 0.229 & 0.188 & $\ldots$ & 0 \\
\hline$\omega_{3}$ & 0 & 0.115 & 0.159 & 0.196 & 0.250 & 0.296 & 0.313 & $\ldots$ & 0 \\
\hline$\omega_{4}$ & 0 & 0.091 & 0.134 & 0.175 & 0.250 & 0.351 & 0.438 & $\ldots$ & 1 \\
\hline$A C I$ & 0.725 & 0.712 & 0.707 & 0.702 & 0.694 & 0.684 & 0.677 & $\ldots$ & 0.635 \\
\hline
\end{tabular}

Assuming a consensus threshold value of $\gamma=0.7$, it is observed that the $A C I$ reaches such consensus threshold when $r=0.74$ and the group of expert would be just at the threshold value of consensus. For a bigger value of $r$ the group would be below the consensus threshold value and the feedback process would be required.

\section{Feedback mechanism based on attitudinal consensus index}

If the $A C I$ is lower than the threshold value, then the feedback mechanism is activated. This paper proposes a feedback mechanism to increase consensus that relies in identifying the elements in the DMs that generate higher discrepancies between individual experts and the group. Once these elements are identified, a set of rules are generated for recommendations (advices) to individual experts on how to modify/adjust the inconsistent elements in their decision matrix with the aim of increasing the group consensus, and keeping the rest of the decision matrix elements unchanged. The attitudinal consensus degree based feedback mechanism for GDM consensus model is shown in Figure 1.

\subsection{Identification of inconsistent decision matrix elements}

This is done in a three-steps process that starts by identifying first the experts in the group with $P D$ lower than the consensus threshold value. This is followed by identifying for these experts the alternatives with $P A$ below the consensus threshold value. Finally, for the identified alternatives, only those decision matrix elements with $P E$ below the consensus threshold value will be targeted for adjustment.

$$
E X P C H=\left\{h \mid A C I<\gamma \wedge P D^{h}<\gamma\right\}
$$




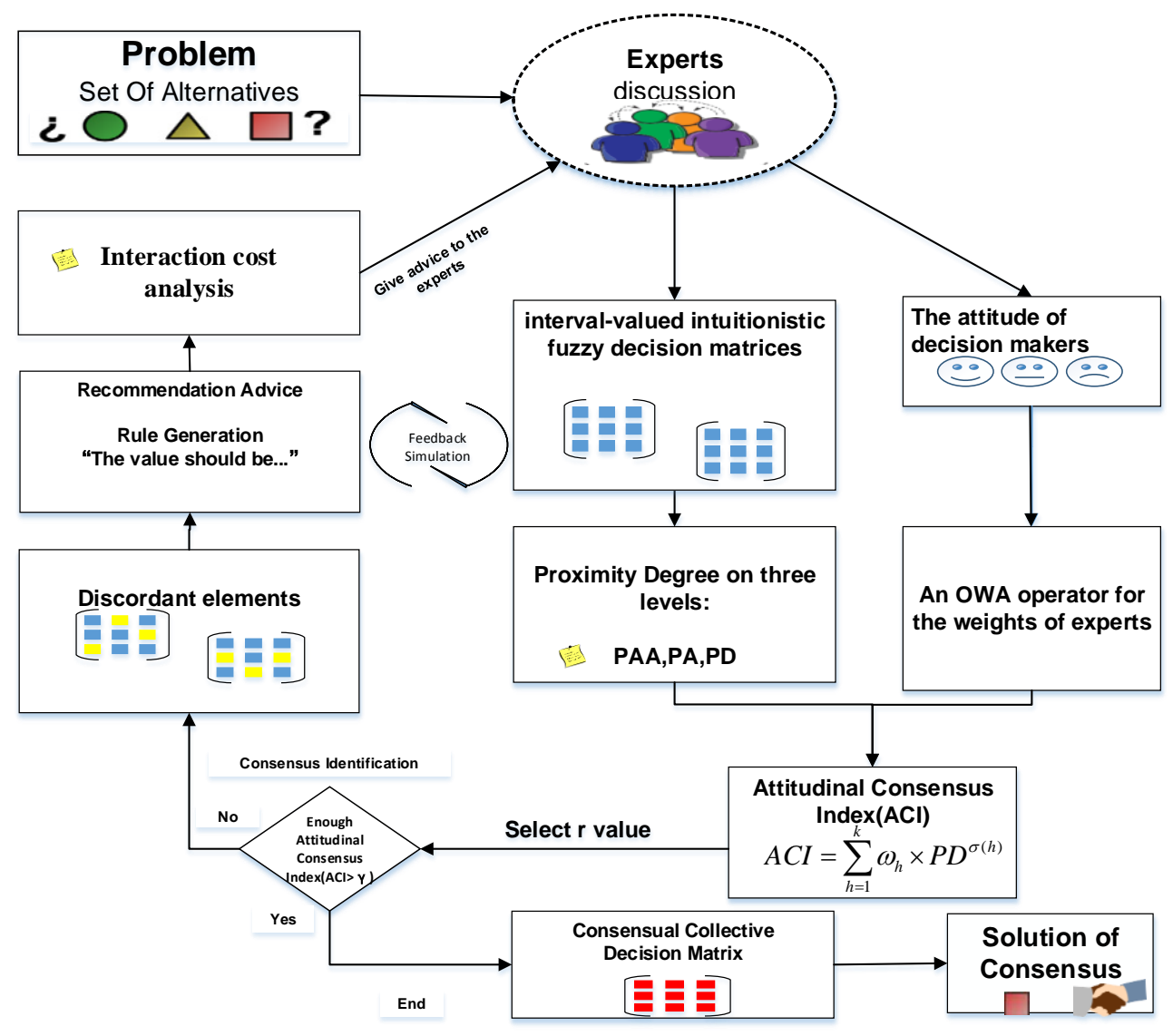

Figure 1: An attitude based feedback mechanism for consensus model for GDM with IFVs

$$
\begin{gathered}
A L T=\left\{(h, i) \mid h \in E X P C H \wedge P A_{i}^{h}<\gamma\right\} \\
A P S=\left\{(h, i, j) \mid(h, i) \in A L T \wedge P E_{i j}^{h}<\gamma\right\}
\end{gathered}
$$

\subsection{Advice generation}

For all $(h, i, j) \in A P S$, the following personalised recommendation rule is defined:

"The evaluation value for the alternatives $\tilde{a}_{i j}^{h}=\left\langle\tilde{u}_{i j}^{h}, \tilde{v}_{i j}^{h}\right\rangle$ should be changed to a value closer to

$$
\tilde{a}_{i j}^{h}(\delta)=\left\langle\tilde{u}_{i j}^{h}(\delta), \tilde{v}_{i j}^{h}(\delta)\right\rangle=\left\langle(1-\delta) \cdot \tilde{u}_{i j}^{h}+\delta \cdot \tilde{u}_{i j}^{c},(1-\delta) \cdot \tilde{v}_{i j}^{h}+\delta \cdot \tilde{v}_{i j}^{c}\right\rangle
$$

where $\delta \in[0,1]$ is a parameter to control the adjustment cost.

\section{Example 3. (Example 2 continuation)}

1. Set of inconsistent decision matrix elements: $A P S=\{(3,2,1),(3,3,2),(3,3,3),(3,4,1),(3,4,3)\}$

2. Taking a value of $\delta=0.3$, the feedback mechanism would provide the following recommendations to expert $e_{3}$ : 
- Your preference value of $\tilde{a}_{21}^{3}$ should be closer to $\langle([0.31,0.41],[0.32,0.42]\rangle$.

- Your preference value of $\tilde{a}_{32}^{3}$ should be closer to $\langle[0.29,0.39],[0.34,0.44]\rangle$.

- Your preference value of $\tilde{a}_{33}^{3}$ should be closer to $\langle[0.36,0.47],[0.49,0.52]\rangle$.

- Your preference value of $\tilde{a}_{41}^{3}$ should be closer to $\langle[0.51,0.61],[0.26,0.36]\rangle$.

- Your preference value of $\tilde{a}_{43}^{3}$ should be closer to $\langle[0.44,0.54],[0.32,0.43]\rangle$.

3. A visual feedback process simulation of current consensus conditions is also shown to the experts (Figure 2). Figure 2(a) depicts the proximity degree on the preference of experts with expert $e_{3}$ being classed as inconsistent, Figure 2(b) shows the proximity degree on alternatives with alternatives 3-5 being inconsistent for $e_{3}$, while Figure 2(c) presents the proximity degree on the elements with elements $a_{32}$ and $a_{33}$ being inconsistent.

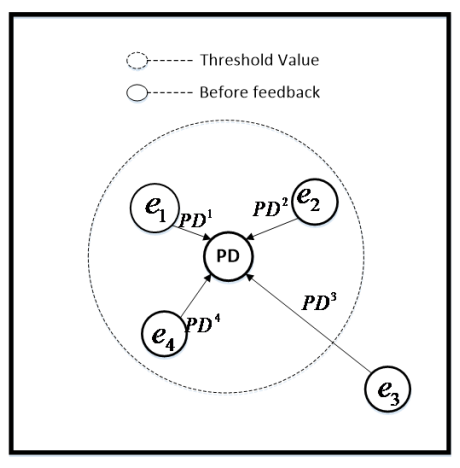

(a) Expert PDs

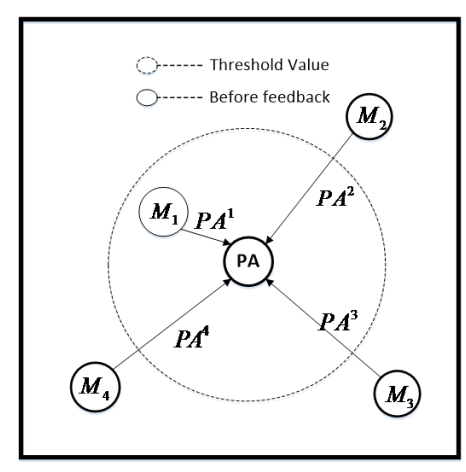

(b) PAs for $e_{3}$

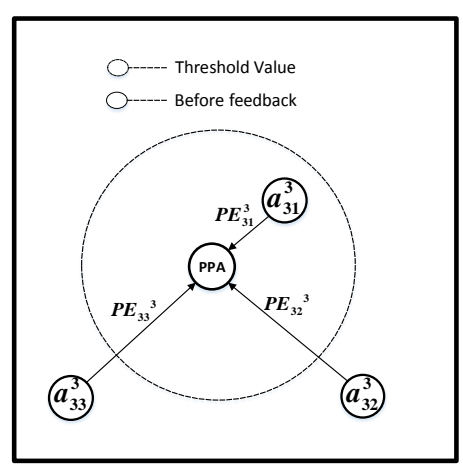

(c) PEs of $M_{3}$ for $e_{3}$

Figure 2: Visual representation of proximity degree at three level.

4. Assuming that expert $e_{3}$ revisits his/her evaluations and implements the recommended IVFNs the new decision matrix would be

$$
A^{3}\left(0.3_{1}\right)=\left(\begin{array}{cccc}
N_{1} & N_{2} & N_{3} \\
M_{1} & \langle[0.20,0.30],[0.30,0.40]\rangle & \langle[0.30,0.40],[0.40,0.50]\rangle & \langle[0.10,0.20],[0.40,0.50]\rangle \\
M_{2} & \langle[0.31,0.41],[0.32,0.42]\rangle & \langle[0.30,0.40],[0.40,0.50]\rangle & \langle[0.50,0.60],[0.40,0.40]\rangle \\
M_{3} & \langle[0.30,0.40],[0.50,0.60]\rangle & \langle[0.29,0.39],[0.34,0.44]\rangle & \langle[0.36,0.47],[0.49,0.52]\rangle \\
M_{4} & \langle[0.51,0.61],[0.26,0.36]\rangle & \langle[0.40,0.50],[0.10,0.30]\rangle & \langle[0.44,0.54],[0.32,0.43]\rangle
\end{array}\right]
$$

- The new PDs are computed:

$$
P D^{1}\left(0.3_{1}\right)=0.731 ; P D^{2}=0.711 ; P D^{3}\left(0.3_{1}\right)=0.642 ; P D^{4}\left(0.3_{1}\right)=0.713
$$

It is noticed here that expert $e_{3}$ still has a PD lower than the assumed consensus threshold value $\gamma=0.7$. 
Table 2: $A C I\left(0.3_{1}\right)$ of the group of the experts

\begin{tabular}{|l|ccccccccc|}
\hline$r$ & 0 & $\frac{1}{3}$ & $\frac{1}{2}$ & $\frac{2}{3}$ & 1 & $\frac{3}{2}$ & 2 & $\ldots$ & $+\infty$ \\
\hline$A C I\left(0.3_{1}\right)$ & 0.731 & 0.718 & 0.712 & 0.707 & 0.699 & 0.690 & 0.683 & $\ldots$ & 0.642 \\
\hline
\end{tabular}

- The new ACI values after this first feedback round are shown in Table 2. In this table, the feedback parameter value we select is 0.3 and the first round of feedback is conducted to obtain the new ACI values:

It can be observed that the threshold consensus value $\gamma=0.7$ is achieved for a value of $r$ close to 1 . Indeed, $\gamma=0.7$ is achieved when $r=0.97$, and therefore the group will reach consensus when $r \leq 0.97$. Otherwise, a second round of feedback would be needed.

5. With feedback parameter $\delta$ still at 0.3 and assuming again that all feedback recommended values are implemented by expert $e_{3}$, then his/her new decision matrix will be

$$
A^{3}\left(0.3_{2}\right)=\left(\begin{array}{cccc}
N_{1} & N_{2} & N_{3} \\
M_{1} & \langle[0.20,0.30],[0.30,0.40]\rangle & \langle[0.30,0.40],[0.40,0.50]\rangle & \langle[0.10,0.20],[0.40,0.50]\rangle \\
M_{2} & \langle[0.31,0.41],[0.33,0.44]\rangle & \langle[0.30,0.40],[0.40,0.50]\rangle & \langle[0.50,0.60],[0.40,0.40]\rangle \\
M_{3} & \langle[0.30,0.40],[0.50,0.60]\rangle & \langle[0.27,0.39],[0.37,0.47]\rangle & \langle[0.33,0.45],[0.49,0.54]\rangle \\
M_{4} & \langle[0.44,0.54],[0.31,0.41]\rangle & \langle[0.40,0.50],[0.10,0.30]\rangle & \langle[0.39,0.49],[0.34,0.45]\rangle
\end{array}\right]
$$

- The new $P D s$ will be:

$$
P D^{1}\left(0.3_{2}\right)=0.735 ; P D^{2}\left(0.3_{2}\right)=0.713 ; P D^{3}\left(0.3_{2}\right)=0.660 ; P D^{4}\left(0.3_{2}\right)=0.718
$$

Again, expert $e_{3}$ increases his/her $\mathrm{PD}$, although it is still below the consensus threshold value of 0.7 .

- The new $A C I$ values are given in Table 3. In this table, the feedback parameter remains unchanged and we conduct the second round of feedback to obtain the new ACI values:

Table 3: $A C I\left(0.3_{2}\right)$ of the group of the experts

\begin{tabular}{|l|ccccccccc|}
\hline$r$ & 0 & $\frac{1}{3}$ & $\frac{1}{2}$ & $\frac{2}{3}$ & 1 & $\frac{3}{2}$ & 2 & $\ldots$ & $+\infty$ \\
\hline$A C I\left(0.3_{2}\right)$ & 0.735 & 0.723 & 0.718 & 0.714 & 0.707 & 0.699 & 0.692 & $\ldots$ & 0.660 \\
\hline
\end{tabular}

We observe that $A C I=\gamma=0.7$ when $r=1.39$, thus a third round of feedback would be needed for $r<1.39$

6. If the third round of feedback is conducted, the new decision matrix of expert $e_{3}$ after imple- 
mented all recommended values with $\delta=0.3$ will be

$$
A^{3}\left(0.3_{3}\right)=\left(\begin{array}{cccc}
N_{1} & N_{2} & N_{3} \\
M_{1} & \langle[0.20,0.30],[0.30,0.40]\rangle & \langle[0.30,0.40],[0.40,0.50]\rangle & \langle[0.10,0.20],[0.40,0.50]\rangle \\
M_{2} & \langle[0.32,0.42],[0.34,0.45]\rangle & \langle[0.30,0.40],[0.40,0.50]\rangle & \langle[0.50,0.60],[0.40,0.40]\rangle \\
M_{3} & \langle[0.30,0.40],[0.50,0.60]\rangle & \langle[0.26,0.38],[0.39,0.49]\rangle & \langle[0.31,0.43],[0.48,0.55]\rangle \\
M_{4} & \langle[0.39,0.49],[0.34,0.44]\rangle & \langle[0.40,0.50],[0.10,0.30]\rangle & \langle[0.36,0.46],[0.35,0.47]\rangle
\end{array}\right]
$$

- The new $P D s$ are computed:

$$
P D^{1}\left(0.3_{3}\right)=0.739 ; P D^{2}\left(0.3_{3}\right)=0.715 ; P D^{3}\left(0.3_{3}\right)=0.711 ; P D^{4}\left(0.3_{3}\right)=0.711
$$

Obviously, after the third round of feedback, we have $P D^{3}\left(0.3_{3}\right)=0.711>\gamma$, and then the inconsistent expert $e_{3}$ is up of the threshold value. Therefore, the group will reach consensus, and then the feedback mechanism is terminated. The PD values of expert $e_{3}$ is now above the threshold consensus value, and it is no longer inconsistent. Therefore, the group will reach consensus, and then the feedback mechanism is terminated. The evolution of the PD of expert $e_{3}$ after each feedback round is shown in Figure 3. It can be seen that the value of PD increases as the number of interactions increases.

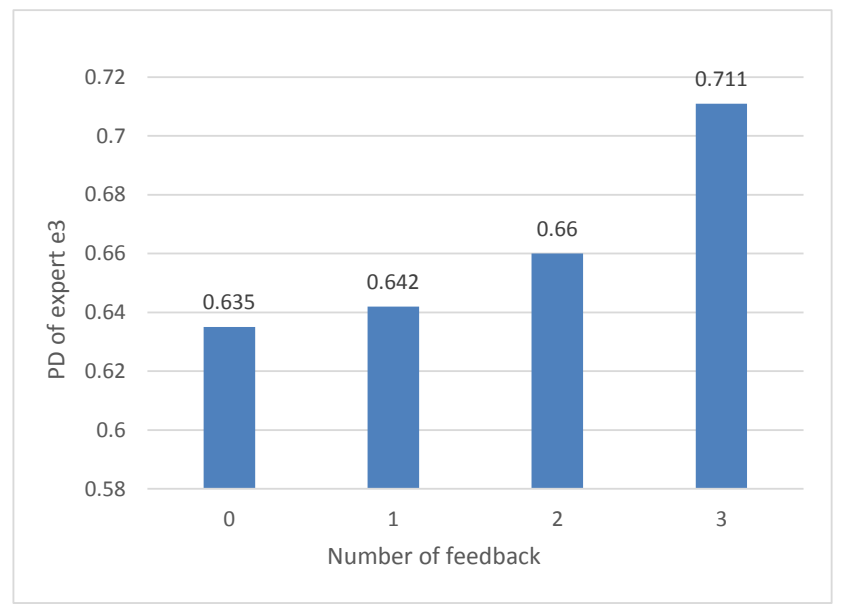

Figure 3: $P D$ value of expert $e_{3}$ before and after feedback

It is now observed that all experts' $P D$ values are above the consensus threshold, which is shown in Figure 4. Figure 4(a) shows the $P D$ values of experts before and (coloured) after the feedback process, with $P D^{3}$ increasing from a value (0.642) lower than to a value (0.711) higher than the assumed consensus threshold value $\gamma=0.7$. Figure $4(\mathrm{~b})$ shows the differences between the $P A$ values for expert $e_{3}$ that were lower than the threshold value $\gamma$ before the feedback and their new (coloured) values after the feedback. Figure 4(c) depicts the change on the $P E$ values for the elements of the decision matrix for expert $e_{3}$ that were lower than the threshold value $\gamma$ before the feedback process and their new (coloured) 
values after the feedback. In this figure the blue solid circles represents PEs after the expert $e_{3}$ feedback.

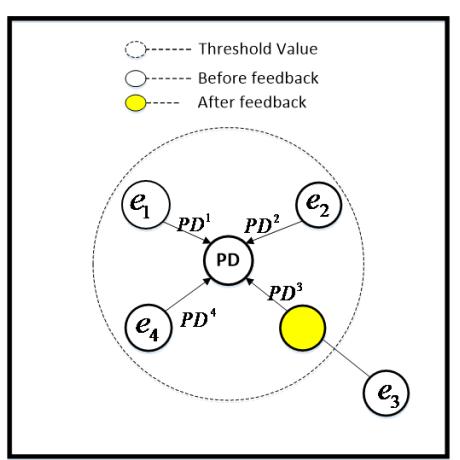

(a) $P D^{3}$ before and after

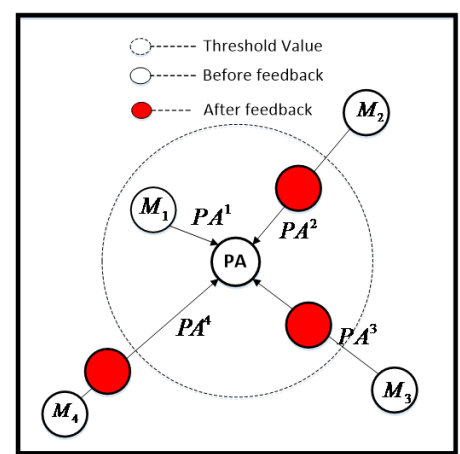

(b) PAs before and after

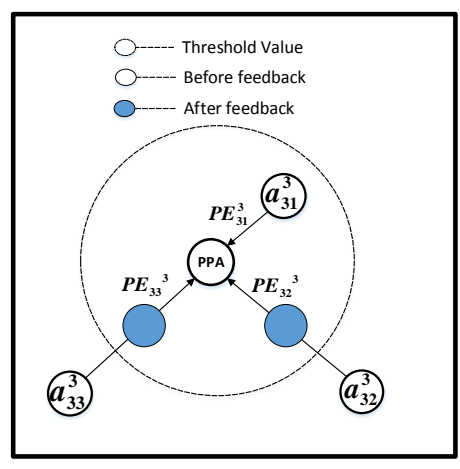

(c) PEs before and after

Figure 4: Simulation of consensus before and after (coloured) feedback recommended values implemented by expert $e_{3}$.

Because the OWA operator is located between the minimum and maximum of the aggregated values, it is clear now that the $A C I$ will be always above the consensus threshold value for all attitudinal parameter values $r$, as are shown below.

- The new $A C I$ values are given in Table 4. In this table, the feedback parameter remains unchanged and we conduct the third round of feedback to obtain the new ACI values:

Table 4: $A C I$ of the group of the experts

\begin{tabular}{|l|ccccccccc|}
\hline$r$ & 0 & $\frac{1}{3}$ & $\frac{1}{2}$ & $\frac{2}{3}$ & 1 & $\frac{3}{2}$ & 2 & $\ldots$ & $+\infty$ \\
\hline$A C I$ & 0.739 & 0.731 & 0.728 & 0.725 & 0.721 & 0.718 & 0.716 & $\ldots$ & 0.711 \\
\hline
\end{tabular}

\section{Adjustment cost analysis with ACI}

This section studies the influence of the attitudinal parameter $r$ on the adjustment costs. Ben-Arieh and Easton [2] provided a very comprehensive analysis of the cost of reaching consensus. Recently, some minimum adjustment cost models for consensus reaching process have been proposed in [16, 21, $22,36,42]$. The adjustments cost of the implementation of the feedback recommendation advices can be defined as [34]:

$$
T C(\delta)=\sum_{h, i, j \in A P S} d\left(\tilde{a}_{i j}^{h}, \tilde{a}_{i j}^{h}(\delta)\right)
$$

\section{Example 4. (Example 3 continuation).}

The adjustment costs at each feedback round of $e_{3}$ are given in Table 5. The total adjustment cost will be $T C(0.3)=T C_{1}(0.3)+T C_{2}(0.3)+T C_{3}(0.3)=1.71$. 


\begin{tabular}{|l|ccc|}
\hline Feedback round & 1 & 2 & 3 \\
\hline$T C_{i}(0.3)$ & 0.720 & 0.558 & 0.432 \\
\hline
\end{tabular}

To visualise the influence of the expert's attitudinal parameter on the adjustment costs, we draw the chart of adjustment cost with different values of attitudinal parameter $r$ in Figure 5. The consensus reaching process can be classified into four steps according to different attitudinal parameter values: (1) If $0 \leq r \leq 0.74$, the group initiates the decision process with enough agreement (consensus), the feedback mechanism is not activated, and no adjustment cost is involved; (2) If $0.74<r \leq 0.97$, there is no initial group consensus and the feedback mechanism is activated and carried out once, with an adjustment cost of 0.720 ; (3) If $0.97<r \leq 1.39$, the feedback process is applied twice, with an additional adjustment cost of 0.558 that brings the total adjustment cost to 1.278; (4) Otherwise, the feedback process is carried out three times with a total adjustment cost of 1.71 .

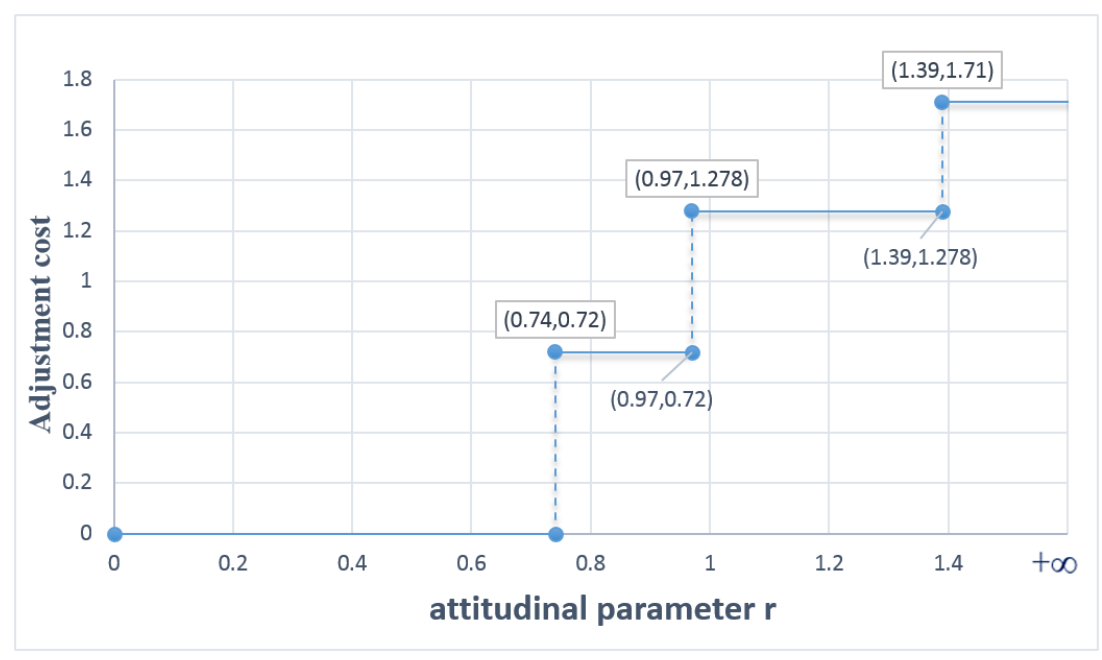

Figure 5: Adjustment cost of $e_{3}$ after each feedback with different attitudinal parameter $r$

\section{Example 5. (Finishing Example 1)}

Without loss of generality, we assume the following attitudinal parameter in the decision scenario $r=5 / 6$ and the following attribute weights of criteria: $\omega=\left(N_{1}: 0.50 ; N_{2}: 0.20 ; N_{3}: 0.30\right)^{T}$. Then the collective overall evaluation values associated to the four alternatives $\left\{M_{1}, M_{2}, M_{3}, M_{4}\right\}$ would result in the following consensus ranking: $M_{4} \prec M_{2} \prec M_{1} \prec M_{3}$, with $r=5 / 6$.

\section{Comparison analysis}

In this section, we conduct a comparison analysis with other traditional policies such as the ' $m i n$ imum disagreement policy' and the 'indifferent disagreement policy' to demonstrate the feasibility of 
our proposed approach.

(i) The 'minimum disagreement policy' [34] used the minimum operator in aggregating individual consensus levels, which in practice requires all the inconsistent experts to reach the consensus threshold value before aggregation. In Figure 5, when parameter $r$ tends to positive infinity, the group uses the 'minimum disagreement policy'. The number of interaction rounds and the adjustment cost of the feedback reach the maximum.

(ii) The 'indifferent disagreement policy' [41] used the arithmetic mean to aggregate individual consensus levels. It can be seen that when $r=1$, the group uses the 'indifferent disagreement policy'. At this point, two rounds of interaction and an increase twofold of the adjustment cost by the feedback process are generated.

(iii) In this paper, a new stop policy is proposed. It is a more general policy because it takes into account group attitudes in a continuous state from pessimistic to optimistic. Both the 'minimum disagreement policy' and the 'indifferent disagreement policy' are special cases of the proposed stop policy as mentioned above. Also, when parameter $r=0$, experts adopt a 'maximum disagreement policy', which is the policy that makes easiest reaching consensus for a group.

\section{Conclusion}

This article proposes an attitudinal consistency function for feedback mechanism in a GDM consensus reaching process. It has the following main advantages and differences with respect to other consensus models introduced in the literature:

(i) It Investigates an attitudinal consensus index (ACI) for consensus reaching process. The advantage of $\mathrm{ACI}$ is that it generally takes into account decision attitudes in a continuous state from pessimistic to optimistic. Therefore, it can be regarded as a general extension approach that extends the 'OWA based policy' [41] and 'pessimistic, indifferent and optimistic' consensus policies [25].

(ii) It builds an ACI based stop policy to control the feedback with different adjustment cost. Since $\mathrm{ACI}$ is a continuous aggregation function (from pessimistic to optimistic), it contains the traditional 'minimum disagreement policy' and 'indifferent disagreement policy' as special cases. Additionally, the adjustment cost is related to the attitudinal parameter in an increasing monotonic way. Indeed, the more pessimistic the attitudinal parameter is the smaller the ACI is, and the higher the adjustment cost will be.

(iii) It was also found that the consensus level of the inconsistent expert will increase after the feedback recommendations are implemented. Using a visual simulation of the change of adjustment cost 
and consensus level with respect to the attitudinal parameter, the inconsistent experts will be able to keep a balance between them by implementing the feedback recommendation advice according to their own willing.

(iv) The feedback parameter of control effect on the consensus reaching process and the adjustment cost is an issue that requires further research for its optimal selection.

\section{Acknowledgements}

The authors thank for the constructive suggestions from the anonymous referees. The authors also are very grateful to Professor Ronald R. Yager for his valuable idea for this article. This work was sponsored by National Natural Science Foundation of China (NSFC) (No.71571166, 71331002, 71503165), Zhejiang Provincial Natural Science Foundation of China (LY16G010005), Zhejiang Provincial Philosophy and Social Sciences Planning Project of China under the Grant (No.13NDJC015Z) and Natural Science Foundation of Shanghai (18ZR1416900).

\section{References}

[1] K. Atanassov, G. Gargov. Interval-valued intuitionistic fuzzy sets, Fuzzy Sets and Systems, 31 (1989) 343-349.

[2] D. Ben-Arieh, T. Easton. Multi-criteria group consensus under linear cost opinion elasticity, Decision Support Systems, 43 (2007) 713-72.

[3] C. Butler, A. Rothstein. On conflict and consensus: A handbook on formal consensus decision making, Takoma Park (2006).

[4] N. Capuano, F. Chiclana, H. Fujita, E. Herrera, V. Loia. Fuzzy group decision making with incomplete information guided by social influence, IEEE Transactions on Fuzzy System, 26 (3) (2018) 1704-1718.

[5] N. Capuano, F. Chiclana, E. Herrera, H. Fujita, V. Loia. Fuzzy rankings for preferences modelling in group decision making, IEEE International Journal of Intelligent Systems, 33 (2018) 1555-1570.

[6] X. Chen, H. Zhang, Y.C. Dong. The fusion process with heterogeneous preference structures in group decision making: A survey, Information Fusion, 24 (2015) 72-83.

[7] J.F. Chu, X. Liu, Y. Wang, K. Chin. A group decision making model considering both the additive consistency and group consensus of intuitionistic fuzzy preference relations, Computers $\&$ Industrial Engineering, 101 (2016) 227-242. 
[8] G. Deschrijver, E. E. Kerre. On the relationship between some extensions of fuzzy set theory, Fuzzy Sets and Systems, 133 (2003) 227-235.

[9] Y. C. Dong, W. Liu, F. Chiclana, E. Herrera-Viedma, F. J. Cabrerizo. Group decision-making based on heterogeneous preference relations with self-confidence, Fuzzy Optimization and Decision Making, 16 (4) (2017) 429-447.

[10] Y. C. Dong, Q. Zha, H. Zhang, G. You, H. Fujita, F. Chiclana, E. Herrera-Viedma Consensus reaching in social network group decision making: Research paradigms and challenges, KnowledgeBased Systems (2018) doi: 10.1016/j.knosys.2018.06.036.

[11] Y. C. Dong, C. C Li, F. Chiclana, E. Herrera-Viedma, Average-case consistency measurement and analysis of interval-valued reciprocal preference relations, Knowledge-Based Systems Volume, 114 (2016) 108-117.

[12] Y.C. Dong, Y. Xu, H. Li. On consistency measures of linguistic preference relations, European Journal of Operational Research, 189 (2008) 430-444.

[13] Y.C. Dong, H. Zhang, E. Herrera-Viedma. Consensus reaching model in the complex and dynamic MAGDM problem, Knowledge-Based Systems, 106 (2016) 206-219.

[14] Y. C. Dong, S. Zhao, H. Zhang, F. Chiclana, E. Herrera-Viedma. A self-management mechanism for non-cooperative behaviors in large-scale group consensus reaching processes. IEEE Transactions on Fuzzy System (2018) 10.1109/TFUZZ.2018.2818078.

[15] Z.W. Gong, X. Xu, H. Zhang, U.A. Ozturk, E. Herrera-Viedma, C. Xu. The consensus models with interval preference opinions and their economic interpretation, Omega-International Journal of Management Science, 55 (2015) 81-90.

[16] Z.W. Gong, H. Zhang, J. Forrest, L. Li, X. Xu. Two consensus models based on the minimum cost and maximum return regarding either all individuals or one individual, European Journal of Operational Research, 240 (2015) 183-192.

[17] E. Herrera-Viedma, F.J. Cabrerizo, J. Kacprzyk, W. Pedrycz. A review of soft consensus models in a fuzzy environment, Information Fusion, 17 (2014) 4-13.

[18] K. Janusz, F. Mario. A soft measure of consensus in the setting of partial (fuzzy) preferences, European Journal on Operational Research, 34 (1988) 316-325.

[19] N. H. Kamis, F. Chiclana, J. Levesley Geo-uninorm consistency control module for preference similarity network hierarchical clustering based consensus model, Knowledge-Based Systems (2018) $706-720$. 
[20] N. H. Kamis, F. Chiclana, J. Levesley Preference similarity network structural equivalence clustering based consensus group decision making model, Applied Soft Computing, 67 (2018) doi: 10.1016/j.knosys.2018.05.039.

[21] Y. Li, H. Zhang, Y.C. Dong. The interactive consensus reaching process with the minimum and uncertain cost in group decision making, Applied Soft Computing, 60 (2017) 202-212.

[22] Y. Liu, Y.C. Dong, H. Liang, F. Chiclana, E. Herrera-Viedma Multiple attribute strategic weight manipulation with minimum cost in a group decision making context with interval attribute weights information, IEEE Transactions on Systems Man Cybernetics: Systems (2018) doi: 10.1109/TSMC.2018.2874942.

[23] Y.J. Liu, C.Y. Liang, F. Chiclana, J. Wu. A trust induced recommendation mechanism for reaching consensus in group decision making, Knowledge-Based Systems, 119 (2017) 221-231.

[24] M. J. del Moral, F. Chiclana, J. M. Tapia, E. Herrera-Viedma. A comparative study on consensus measures in group decision making, International Journal of Intelligent Systems, 33 (2018) 16241638.

[25] I. Palomares, R.M. Rodríguez, L. Martínez. An attitude-driven web consensus support system for heterogeneous group decision making, Expert Systems with Applications, 50 (2013) 139-149.

[26] I.J. Perez, F.J. Cabrerizo, S. Alonso, Y .C. Dong, F. Chiclana, E. Herrera-Viedma On dynamic consensus processes in group decision making problems, Information Sciences, 459 (2018) 20-35.

[27] L. G. Perez, F. Mata, F. Chiclana, G. Kou, E, Herrera-Viedma. Modelling influence in group decision making, Soft Computing, 20 (4) (2016) 1653-1665.

[28] S. Saint, J.R. Lawson. Rules for reaching consensus: A modern approach to decision making, John Wiley $\&$ Sons (1994).

[29] R. Ureña, F. Chiclana, G. Melancon, E. Herrera-Viedma. A social network based approach for consensus achievement in multiperson decision making, Information Fusion Volume, 47 (2019) $72-87$.

[30] J. Wu and F. Chiclana. Non-dominance and attitudinal prioritisation methods for intuitionistic and interval-valued intuitionistic fuzzy preference relations, Expert Systems with Applications, 39 (2012) 13409-13416.

[31] J. Wu, F. Chiclana, H. Fujita, E. Herrera-Viedma. A visual adjustment consensus model for social network group decision making with trust propagation, Knowledge-Based Systems, 122 (2017) 3950. 
[32] J. Wu, F. Chiclana, E. Herrera-Viedma. Trust based consensus model for social network in an incomplete linguistic information context, Applied Soft Computing, 35 (2015) 827-839.

[33] J. Wu, F. Chiclana, H.C. Liao. Isomorphic multiplicative transitivity for intuitionistic and interval-valued fuzzy preference relations and its application in deriving their priority vectors, IEEE Transactions on Fuzzy Systems, 26 (2018) 193-202.

[34] J. Wu, L.F. Dai, F. Chiclana, H. Fujita, E. Herrera-Viedma. A minimum adjustment cost feedback mechanism based consensus model for group decision making under social network with distributed linguistic trust, Information Fusion, 41 (2018) 232-242.

[35] J. Wu, R. Xiong, F. Chiclana. Uninorm trust propagation and aggregation methods for group decision making in social network with four tuples information, Knowledge-Based Systems, 96 (2016) 29-39.

[36] Z.B. Wu, J.P. Xu. A consensus model for large-scale group decision making with hesitant fuzzy information and changeable clusters, Information Fusion, 41(2018)217-231.

[37] Y.J. Xu, X. Wen, W. Zhang. A two-stage consensus method for large-scale multi-attribute group decision making with an application to earthquake shelter selection, Computers $\mathcal{E}$ Industrial Engineering, 116 (2018) 113-129.

[38] Z.S. Xu. Methods for aggregating interval-valued intuitionistic fuzzy information and their application to decision making, Control and Decision, 22 (2007)215-219.

[39] Z.S. Xu, R.R. Yager. Intuitionistic and interval-valued intutionistic fuzzy preference relations and their measures of similarity for the evaluation of agreement within a group, Fuzzy Optimization and Decision Making, 8 (2009) 123-39.

[40] R.R. Yager. Quantifier guided aggregation using OWA operators, International Journal of Intelligent Systems, 11 (1996) 49-73.

[41] R.R. Yager, N. Alajlan. An intelligent interactive approach to group aggregation of subjective probabilities, Knowledge-Based Systems, 83 (2015) 170-175.

[42] B.W. Zhang, Y.C. Dong, Y. Xu. Multiple attribute consensus rules with minimum adjustments to support consensus reaching, Knowledge-Based Systems, 67 (2014) 35-48.

[43] G.Q. Zhang, Y.C. Dong, Y. Xu. Consistency and consensus measures for linguistic preference relations based on assignation assessments, Information Fusion, 17 (2014) 46-55. 